\title{
Calculation of Ground and Excited State Polarizabilities of Unsubstituted and Donor/ Acceptor Polyenes: A Comparison of the Finite-Field and Sum-Over-States Methods ${ }^{\S}$
}

\author{
Sarah A. Locknar, ${ }^{\dagger}$ Linda A. Peteanu, ${ }^{*}, \dagger$ and Zhigang Shuai ${ }^{*}$ \\ Department of Chemistry, Carnegie Mellon University, Pittsburgh, Pennsylvania 15213, and \\ Center for Research on Molecular Electronics and Photonics, Université de Mons-Hainaut, \\ Place du Parc 20, B-7000 Mons, Belgium
}

Received: July 23, 1998; In Final Form: September 30, 1998

\begin{abstract}
In this manuscript, a relatively simple and inexpensive INDO/SCI finite-field (FF) method for calculating polarizabilities $(\alpha)$ is demonstrated to give good agreement with results obtained by both the INDO/MRD/ SDCI sum-over-states routine and published results using the RPA method. The FF method is as effective as the other techniques in predicting both ground and excited state $\alpha$ 's in all substituted and unsubstituted polyenes studied. We observe the correlation described by Marder and co-workers between bond-order-alternation (BOA) and dipolar properties, such as the change in $\alpha$ between the ground and excited states $(\Delta \alpha)$. In addition, qualitative, but not quantitative, agreement is seen between the calculated $\Delta \alpha$ 's of polar polyenes and those measured by Stark-effect spectroscopy.
\end{abstract}

Recent interest in substituted polyenes which exhibit nonlinear optical (NLO) behavior has made accurate calculations of their properties an important predictive tool in materials development. For example, the hyperpolarizabilities $(\beta)$ of molecules are of interest because this parameter is directly related to efficiency in optical frequency doubling and to the Pockel effect. Experimentally, there are two common strategies for optimizing $\beta:{ }^{1-7}$ (1) changing the donor (D) and/or acceptor (A) strength of substituents on the polyene and (2) changing the solvent polarity and/or polarizability. Both alter the local field of the polyene chain, leading to a change in bond-order alternation $(\mathrm{BOA})^{8}$ and in NLO properties, including $\beta$. In the two-state model, ${ }^{9,10}$ $\beta$ is proportional to the change in dipole moment between the ground and excited states $(\Delta \mu)$, so an accurate calculation of solvated $\Delta \mu$ is needed to correctly model the effects of solvation on $\beta{ }^{3,6,11-16}$ In dielectric cavity models, ${ }^{17}$ the solvent-corrected $\Delta \mu$ also depends on the change in polarizability between the ground and excited states $(\Delta \alpha)$. Therefore, quantitatively accurate calculations of both ground and excited state $\alpha$ are needed to correctly model the solvent-effects on $\beta$ and $\Delta \mu$, particularly in highly polarizable systems such as the substituted polyenes. This paper compares the two most common routines for calculating ground state $\alpha$, the sum-over-states and finite-field methods, and applies them to the calculation of excited state $\alpha$.

While techniques for the calculations of first $(\beta)$ and second $(\gamma)$ order hyperpolarizabilities and ground-state polarizabilities $\left(\alpha_{\mathrm{g}}\right)$ of polyenes are well developed, ${ }^{12,14,15,18-23}$ we are aware of only one example of excited-state polarizability $\left(\alpha_{\mathrm{e}}\right)$ calculations for such systems in the literature. ${ }^{24}$ In this published work, the random phase approximation (RPA) was utilized to investigate a series of linear, unsubstituted polyenes. Marder and coworkers have investigated trends in $\Delta \alpha^{25}$ and $\alpha_{\mathrm{g}}{ }^{1}$ with respect to BOA, but to our knowledge, no quantitative calculations of $\alpha_{\mathrm{e}}$ or $\Delta \alpha$ have been published on substituted systems similar to those being developed for NLO applications. Because linear

\footnotetext{
$\dagger$ Department of Chemistry.

Center for Research on Molecular Electronics and Photonics.

$\S$ This paper is dedicated to the memory of Professor Bryan Kohler, whose work continues to be an inspiration.
}

unsubstituted polyenes have been extensively studied in an attempt to learn more about the properties of longer systems, ${ }^{19,26,27}$ the first part of this paper will focus on the linear (long-axis) $\alpha$ calculations in polyenes. The second part investigates the calculation of $\alpha_{e}$ and $\Delta \alpha$ as a function of D/A strength in a series of substituted diphenylbutadienes and other selected D/A polyenes.

We shall compare two techniques for calculating $\alpha_{\mathrm{g}}$ and $\alpha_{\mathrm{e}}$ in polyenes in which identical geometries and semiempirical methods (INDO1S ${ }^{28,29}$ ) are used. The first method includes only single excitations from the Hartree-Fock (HF) ground state (single configuration interaction - SCI), while the second method includes a multireference-determinant/single double configuration interaction (MRD/SDCI), and therefore includes all single and double excitations along with a subset of triple and quadruple excitations. These two methods generate the electronic energy levels of the molecule. Once generated, we employ one of two techniques to calculate the actual polarizabilities: the finite-field (FF) method ${ }^{30-32}$ with INDO/SCI and the sum-over-states (SOS) method ${ }^{20}$ with INDO/MRD/SDCI. The FF method calculates the perturbation in the energies and dipole moments of the energy levels as the result of applying an electric field $(\mathrm{F})$, from which $\alpha$ can be calculated as

$$
\alpha=\frac{\mu_{\mathrm{F}}-\mu_{\mathrm{o}}}{\mathrm{F}}
$$

where $\mu_{\mathrm{F}}$ and $\mu_{\mathrm{o}}$ represent the dipole moments in the presence and absence of the field, respectively. In the SOS method, $\alpha$ is obtained from perturbation theory as

$$
\alpha=\sum_{i \neq f} \frac{|\langle f|m| i\rangle|^{2}}{E_{f}-E_{i}}
$$

where $i$ is the state of interest, $f$ represents all other states, and $m$ is the transition moment between the states. In the past, we have used the INDO1S/SCI/FF method to calculate $\Delta \alpha$ in retinals and their Schiff base ${ }^{33}$ and the INDO1S/MRD/SDCI/ 
SOS method to calculate the NLO properties of both oligomer and $\mathrm{D}-\mathrm{A}$ polyene systems. ${ }^{34,35}$

Methods. Ground-state geometries of the diphenylpolyenes were optimized using the $\mathrm{AM} 1^{36}$ molecular orbital procedure $\left(M_{0 p a c}^{37}\right)$. To facilitate comparisons to literature values, we have used idealized geometries for the linear, unsubstituted polyenes $^{24}$ (bond lengths in angstroms: $(\mathrm{C}-\mathrm{C})=1.443,(\mathrm{C}=$ $\mathrm{C})=1.366,(\mathrm{C}-\mathrm{H})=1.085$. Bond angles: $(\mathrm{C}-\mathrm{C}-\mathrm{H})=118.1$, $\left.(\mathrm{C}-\mathrm{C}=\mathrm{C})=123.8^{38}\right)$. Identical geometries were used for both SOS and FF methods. For the FF calculations, ${ }^{30,31}$ the polarizability was obtained from the field dependence of the dipole moments generated from INDO1S/SCI ${ }^{28,29}$ as described above. The active space for INDO1S/SCI/FF calculations was five occupied and five virtual MOs, and the Mataga-Nishimoto potential was used..$^{39,40} \mathrm{We}$ found that the properties of interest converged with the number of states mentioned above, so inclusion of additional states was not necessary. Because the FF method depends only on the dipole moment of the state of interest, SCI should be sufficient to describe $\alpha_{e}$, much as Hartree-Fock/FF is adequate for calculating $\alpha_{\mathrm{g}}$.

A modified version of MRD/SDCI, implemented in the semiempirical quantum chemical ZINDO package, ${ }^{28,29}$ was used for the SOS calculations. Given the nature of the SOS calculation, a good description of two-photon states is needed to properly calculate $\alpha$ of the $B_{u}$ state. Within MRD/SDCI, a normal SDCI calculation is executed to find reference determinants for the most important low-lying excited states. Usually there are six of these, namely, (1) Hartree-Fock ground state, (2) $\mathrm{H} \rightarrow \mathrm{L}$ (HOMO $\rightarrow$ LUMO) single excitation, (3) $\mathrm{H} \rightarrow \mathrm{L}+1$ single, (4) $\mathrm{H}$ $-1 \rightarrow \mathrm{L}$ single, (5) $\mathrm{HH} \rightarrow \mathrm{LL}$ double, and $(6)(\mathrm{H}-1) \mathrm{H} \rightarrow \mathrm{L}(\mathrm{L}+1)$ double. On the basis of these references, we then perform SDCI so that the result contains many triple and quadruple excitations. As shown by Tavan and Schulten, ${ }^{41,42}$ this approach largely removes the size inconsistency problem of normal SDCI. All the configurations are spin-adapted via valence bond diagrams. The Ohno-Klopman potential ${ }^{43,44}$ is used in order to reproduce the relative ordering of the $1 \mathrm{~B}_{\mathrm{u}}$ and $2 \mathrm{~A}_{\mathrm{g}}$ states. This approach has been applied by Shuai, Beljonne, and Brédas ${ }^{34,35}$ to calculate NLO properties of polyenes and oligothiophenes and has been shown to provide reasonable agreement with experimental values. ${ }^{34,35}$ Six occupied and six virtual MOs are active orbitals, amounting to 703 spin-adapted configurations for SDCI and 3275 configurations for MRD/SDCI. The Hamiltonian matrix is then diagonalized for the lowest 800 states to calculate the transition moments and eventually to calculate the polarizabilities both for the ground state and for the lowest optically allowed excited state. From this point onward, the SDCI abbreviation for MRD/SDCI will be used, and unless otherwise noted, reported values for $\alpha$ are averaged over all three directions.

\section{Results and Discussion}

Unsubstituted Linear Polyenes. To compare our results with recently published random phase approximations (RPA) calculations, ${ }^{24,45}$ the values of $\alpha$ and energy were fit to a power law with respect to the number of repeat units $n$. The RPA ${ }^{46}$ method can be obtained from Green's function theory. The linear response $\alpha$ from RPA should be identical to that obtained from the SCI/FF method ${ }^{46,47} \mathrm{We}$ shall begin by comparing the SCI/ $\mathrm{FF}, \mathrm{SDCI} / \mathrm{SOS}$, and RPA results.

In Figure 1a the long-axis $\alpha$ 's of the $1 \mathrm{~A}_{\mathrm{g}}$ and $1 \mathrm{~B}_{\mathrm{u}}$ states from the SCI/FF and SDCI/SOS calculations are shown together with RPA results and their power law fits. Similarly, in Figure $1 b$ the calculated and measured energy gaps between the $1 \mathrm{~A}_{\mathrm{g}}$ and

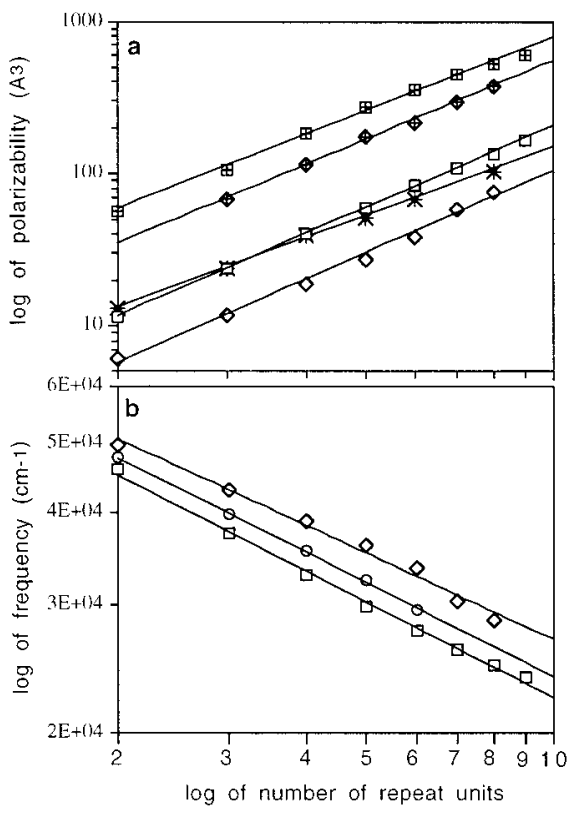

Figure 1. (a) Polarizabilities along the long axis of a series of polyenes calculated with the FF and RPA methods. Squares signify calculations done with the SCI/FF method (unfilled is the $1 \mathrm{~A}_{\mathrm{g}}$ ground state and crossed is the $1 \mathrm{~B}_{\mathrm{u}}$ state). The curves are described by $\alpha_{\mathrm{Bu}}=(19.32)$ $n^{1.60}$ and $\alpha_{\mathrm{Ag}}=(3.34) n^{1.79}$ where $n$ is the number of repeat units. The asterisks are results of an RPA calculation of the $1 \mathrm{~A}_{\mathrm{g}}$ in the literature ${ }^{45}$ $\left(\alpha_{\mathrm{Ag}}=(4.61) n^{1.51}\right)$. Diamonds represent the SDCI/SOS results (unfilled is the $1 \mathrm{~A}_{\mathrm{g}}$ ground state, and crossed is the $1 \mathrm{~B}_{\mathrm{u}}$ state). These curves are described by $\alpha_{\mathrm{Bu}}=(10.39) n^{1.72}$ and $\alpha_{\mathrm{Ag}}=(1.63) n^{1.80}$. (b) Energies for $1 \mathrm{~B}_{\mathrm{u}} \leftarrow 1 \mathrm{~A}_{\mathrm{g}}$ transition calculated by the SDCI/SOS (diamonds, frequency $=66405 n^{-0.40}$ ) and SCI/FF (squares, frequency $=61016 n^{-0.44}$ ) method. Circles are experimental values ${ }^{48}$ (frequency $=64190 n^{-0.43}$, where $n$ is the number of repeat units).

$1 \mathrm{~B}_{\mathrm{u}}$ states are plotted with experimental values. ${ }^{48}$ First comparing the SCI/FF and SDCI/SOS calculations, we see that the power-law fits for the two are nearly parallel for the calculations of $\alpha$, with the SCI/FF results approximately a factor of 2 larger in all cases. We also observe stabilization of the ground-state energy relative to SCI when DCI is included. It becomes evident in Figure $1 \mathrm{~b}$ that the energy gap between the $1 \mathrm{~A}_{\mathrm{g}}$ and $1 \mathrm{~B}_{\mathrm{u}}$ states is overestimated using the SDCI/SOS method, and the transition moment $(m)$ is underestimated (not shown). Choice of potential in the two methods may contribute to these energy differences. In the SCI/FF method, the Mataga-Nishimoto potential is used which is optimized for fitting the lowest optically allowed transition $\left(1 \mathrm{~B}_{\mathrm{u}} \leftarrow 1 \mathrm{~A}_{\mathrm{g}}\right)$, while the Ohno-Klopman potential is used in the SDCI/SOS method. The latter is chosen to correctly predict the ordering of the $1 \mathrm{~B}_{\mathrm{u}}$ and $2 \mathrm{~A}_{\mathrm{g}}$ states but, as a result, the energy of the $1 \mathrm{~B}_{\mathrm{u}} \leftarrow 1 \mathrm{~A}_{\mathrm{g}}$ transition is overestimated. Because $\alpha$ depends linearly on $m$ and inversely on the energy gap, it is not suprising that $\alpha$ is lower when SDCI-SOS is used. However, as shown below, these effects cancel when calculating the change in $\alpha$ between the $1 \mathrm{~A}_{\mathrm{g}}$ and $1 \mathrm{~B}_{\mathrm{u}}$ states.

Comparing our results to those obtained using RPA in the literature ${ }^{45}$ the $1 \mathrm{Ag}$ state shows a power law dependence of $\alpha$ $\propto n^{1.8}$ using both the SCI/FF and SDCI/SOS methods and $\alpha \propto$ $n^{1.51}$ with RPA. ${ }^{45}$ Interestingly, however, in a later work using RPA, a power law dependence of $\alpha \propto n^{1.7}$ was determined, which is much closer to the values obtained in this work. ${ }^{24}$ The percent deviation between RPA and SCI/FF values of $\alpha$ for $n$ $=8$ is $27 \%$ and deviations for shorter repeat units are smaller. Our results also agree with those in the literature which used a perturbative expansion of the density matrix technique and found $\alpha \propto n^{1.75}$. 49 The power law observed for the $1 \mathrm{~B}_{\mathrm{u}}$ state is 
TABLE 1: Polarizability Calculations on Diphenylpolyenes ${ }^{a}$

\begin{tabular}{|c|c|c|c|c|c|c|}
\hline & \multicolumn{3}{|c|}{$\alpha_{\mathrm{g}}$} & \multicolumn{3}{|c|}{$\Delta \alpha$} \\
\hline & SCI-FF & SDCI-SOS & literature & SCI-FF & SDCI-SOS & literature \\
\hline DPB & 25 & 14 & $44^{a}$ & 55 & 48 & $\begin{array}{l}28^{b} \\
27^{c}\end{array}$ \\
\hline DPH & 33 & & & 69 & & $42^{c}$ \\
\hline DPO & 40 & 18 & $49^{b}$ & 86 & 74 & $\begin{array}{l}80^{b} \\
57^{c}\end{array}$ \\
\hline DPD & 45 & & & 94 & & $70^{c}$ \\
\hline
\end{tabular}

${ }^{a}$ All polarizabilities are reported in $\AA^{3}$ and are the average over all directions. Abbreviations: DPB-diphenylbutadiene, DPH-diphenylhexatriene, DPO-diphenyloctatetraene, DPD-diphenyldecapentaene. ${ }^{b}$ See ref $60 .{ }^{c}$ See ref 61 .

$\alpha \propto n^{1.6}$ with SCI/SOS, $\alpha \propto n^{1.7}$ with SDCI/SOS, and $\alpha \propto n^{1.8}$ with $\mathrm{RPA}^{24}$ (raw data not available). Considering the variability in the power law of the ground state using the RPA method described above, the agreement among these three methods is acceptable. ${ }^{50} \mathrm{We}$ also see good agreement in the power laws describing the $1 \mathrm{~B}_{\mathrm{u}} \leftarrow 1 \mathrm{~A}_{\mathrm{g}}$ transition energies (Figure 1b: $\mathrm{E} \propto$ $n^{-0.44}$ for SCI/FF, $\mathrm{E} \propto n^{-0.40}$ for SDCI/SOS, and $\mathrm{E} \propto n^{-0.43}$ for experimental). The absolute magnitudes of SCI/FF calculations and experiment differ by only about $2200 \mathrm{~cm}^{-1}$ on average. The above comparison demonstrates that both the SCI/FF and SDCI/SOS methods are at least as accurate as RPA for describing $\alpha$ of the ground and first excited states of the unsubstituted linear polyenes.

Comparison to Experiment. A common method used to measure $\Delta \alpha$ experimentally is Stark-effect (electroabsorption) spectroscopy. ${ }^{51,52}$ This method measures the perturbation of the absorption spectrum as a function of a large $\left(\sim 10^{6} \mathrm{~V} / \mathrm{cm}\right)$ applied electric field. Unsubstituted diphenylpolyenes have been studied using this technique and comparisons between the calculated and measured values are seen in Table 1 (reproduced from an earlier work ${ }^{33}$ ). We see better agreement between the $\mathrm{SCI} / \mathrm{FF}$ results and experiment with increasing chain length. The SDCI/SOS method gives much smaller values for $\alpha_{g}$ by comparison to SCI/SOS, for which $\alpha_{\mathrm{g}}$ lies within $20 \%$ of SCIFF results (not shown). Apparently, $\alpha_{\mathrm{g}}$ is lowered by dynamic correlation effects that are included by mixing in doubly excited configurations into the ground state. We note, however, that the change in polarizability between the ground $\left(1 \mathrm{~A}_{\mathrm{g}}\right)$ and the $1 \mathrm{~B}_{\mathrm{u}}$ states $(\Delta \alpha)$ obtained at the SCI level is nearly equal to that obtained at the SDCI level. Thus, it appears that the dynamic correlation included at the MRD/SDCI level affects the $1 \mathrm{~A}_{\mathrm{g}}$ and $1 \mathrm{~B}_{\mathrm{u}}$ states to a similar extent. (The MRD/SDCI method used here contains dynamic correlation in both the $1 \mathrm{~A}_{\mathrm{g}}$ and $1 \mathrm{~B}_{\mathrm{u}}$ states since it includes all single and double excitations from both the HF ground state and from the configurations that dominate the $1 \mathrm{~B}_{\mathrm{u}}$ state.)

Substituted Polyenes. Although the agreement between experimental and calculated values of $\Delta \alpha$ in unsubstituted polyenes is good, Stark-effect measurements of $\Delta \alpha$ on substituted polyenes show large deviations from the calculated values, ${ }^{53,54}$ as discussed below. To understand this phenomenon, the effects of substitution on $\Delta \alpha$ have been investigated. For this study, we have chosen para-substituted diphenylbutadiene (DPB) as our model. Both $\Delta \alpha$ and $\Delta \mu$ for the molecules and the ground-state values are shown in Table 2. As expected, inclusion of DCI does not appear to significantly affect $\mu_{\mathrm{g}}$ which is the result of the expectation value obtained from the $\mathrm{CI}$ calculation. Values of $\Delta \mu$ and $1 \mathrm{~B}_{\mathrm{u}} \leftarrow 1 \mathrm{~A}_{\mathrm{g}}$ transition energy only differed significantly between the two methods when a nitro group was included. In the nitro compounds, the calculated frequency difference between the SCI and SDCI methods is
$3500 \mathrm{~cm}^{-1}$ while in the other five substituted molecules, the average deviation is only about $2600 \mathrm{~cm}^{-1}$.

As seen above in the symmetric polyenes, $\alpha_{\mathrm{g}}$ calculated using $\mathrm{SDCI} / \mathrm{SOS}$ is much lower than using SCI/FF, while $\Delta \alpha$ using $\mathrm{SDCI} / \mathrm{SOS}$ is very close to that calculated using SCI/FF (Table 2). Once again, we observe that the SCI method more accurately predicts the energy of the $1 \mathrm{~B}_{\mathrm{u}} \leftarrow 1 \mathrm{~A}_{\mathrm{g}}$ transition because addition of DCI tends to lower the ground state energy. It is likely that the deviations between the measured energies and calculated ones can be attributed to solvent stabilization. Using the Onsager model ${ }^{55}$ we estimate the extent of energy stabilization to be greater than $1500 \mathrm{~cm}^{-1}$.

Effect of BOA. The magnitudes of $\Delta \mu, \Delta \alpha, \alpha_{\mathrm{g}}$, and the nonlinear optical properties of $\beta$ and $\gamma$ correlate to the BOA of the polyene as described by Marder and co-workers. ${ }^{2,25}$ For example, as the BOA approaches zero (the cyanine limit), $\Delta \mu$ approaches zero, $\alpha_{\mathrm{g}}$ approaches its maximum value, and $\Delta \alpha$ approaches its minimum (negative) value. Motivated by the success of this relationship in predicting geometry-related changes in the dipolar properties of polyenes, we investigated the effect of D/A substitution on the BOA of the systems studied here. Interestingly, introduction of D/A groups to the DPB molecule has little effect on $\alpha_{\mathrm{g}}, \Delta \alpha$, or BOA. Only when the groups get very large (as when both a nitro and dimethylamino groups are present) do we see a significant change in $\Delta \alpha$. In the series of substituted molecules studied here, the BOA changed less than $3 \%$ (from -0.571 in DPB to -0.556 in dimethylamino-nitroDPB) across the entire series and we observed a slight increase in $\Delta \alpha$. On the basis of the model proposed by Marder, ${ }^{1,2,54}$ when BOA shifts toward zero, $\Delta \alpha$ should decrease. In general, inclusion of phenyl groups tends to decrease the D-A strength of substituents ${ }^{25,56}$ and would consequently result in smaller changes in BOA. Therefore, we examined the AM1 optimized geometries and FF generated $\Delta \alpha$ 's of substituted decapentaene (which has a similar $\alpha_{\mathrm{g}}$ to DPB). In this molecule, the BOA changed $16 \%$ (from -0.568 in decapentaene to -0.485 in 1-(dimethylamino)-10-nitrodecapentaene) and $\Delta \alpha$ decreased from $67 \AA^{3}$ in the unsubstituted to $24 \AA^{3}$ in the substituted species, in agreement with Marder's model.

To compare to the results of Meyers et al. ${ }^{1}$ which examined the influence of external fields (such as those present in solvent cavities $^{53}$ ) on the BOA and other properties of polyenes using the INDO/SDCI/SOS method, we investigated $\alpha_{\mathrm{g}}$ and $\Delta \mu$ on 1-(dimethylamino)-nonatetraen-9-al (DAO) with the FF method, using two different values of BOA (Table 3, bond lengths given directly in ref 1). The first (smaller) BOA corresponds to the molecule in the gas phase without an applied field, while the second corresponds to a near zero BOA with an applied field of $7.5 \times 10^{10} \mathrm{~V} / \mathrm{cm}$. In general, we see good agreement between the two methods, especially in the trends relating BOA, $\alpha_{\mathrm{g}}$, and $\Delta \mu$. The slight differences observed could be due to small variations in torsion or bond angles used in the two studies. ${ }^{1}$

Comparison to Experimental Values. We have also calculated $\Delta \alpha$ and $\Delta \mu$ for two $\mathrm{D}-\mathrm{A}$ polyenes which were found to have very large values of $\Delta \alpha$ using Stark-effect spectroscopy. ${ }^{53,54}$ The first of these (1-(dimethylamino)-heptatrien-7al, AHTA) showed good agreement with the electroabsorption data for both the BOA and $\Delta \mu$ values, but not for $\Delta \alpha$ (Table $3)$. The striking discrepancy between calculated and measured values of $\Delta \alpha$, compared to that of $\Delta \mu$, has also been observed in our group in the case of the retinals. ${ }^{33}$ In the second molecule, 1,1-dicyano-6-(dimethylamino)-hexatriene (CAHT), the AM1 optimized geometry yielded a much larger BOA than predicted 
TABLE 2: Calculations on Substituted DPB Using SDCI-SOS and SCI-FF Methods

\begin{tabular}{|c|c|c|c|c|c|c|c|c|c|c|c|c|c|c|c|c|}
\hline \multirow[b]{2}{*}{ donor } & \multirow[b]{2}{*}{ acceptor } & \multicolumn{3}{|c|}{$\alpha_{\mathrm{g}}\left(\AA^{3}\right)$} & \multicolumn{2}{|c|}{$\overline{\Delta \alpha}\left(\AA^{3}\right)$} & \multicolumn{3}{|c|}{$\mu_{\mathrm{g}}(\mathrm{D})$} & \multicolumn{2}{|c|}{$\Delta \mu(\mathrm{D})$} & \multicolumn{2}{|c|}{$m(\mathrm{D})$} & \multicolumn{3}{|c|}{$v\left(\mathrm{~cm}^{-1}\right)$} \\
\hline & & SDCISOS & SCI-FF & $\overline{\operatorname{lit}^{a}}$ & SDCISOS & $\overline{\text { SCI-FF }}$ & $\overline{\mathrm{SDCI}}$ & SCI & literature $^{a}$ & $\overline{\mathrm{SDCI}}$ & $\overline{\mathrm{SCI}}$ & $\overline{\mathrm{SDCI}}$ & $\overline{\mathrm{SCI}}$ & $\overline{\mathrm{SDCI}}$ & SCI & $\overline{\text { literature }}$ \\
\hline $\mathrm{H}$ & $\mathrm{H}$ & 14 & 25 & $44^{b}$ & 48 & 55 & & & & & & 9.2 & 10.8 & 34360 & 31430 & $30801^{c}$ \\
\hline $\mathrm{H}_{3} \mathrm{CO}$ & $\mathrm{H}$ & 14 & 27 & & 48 & 56 & 1.7 & 1.8 & & 0.7 & 0.2 & 9.2 & 11.0 & 33720 & 30700 & $30120^{d, e}$ \\
\hline $\mathrm{Me}_{2} \mathrm{~N}$ & $\mathrm{H}$ & 15 & 29 & & 54 & 57 & 1.8 & 1.8 & & 3.2 & 2.9 & 9.5 & 11.2 & 32590 & 30110 & \\
\hline $\mathrm{H}$ & $\mathrm{CN}$ & 16 & & & 55 & 59 & 5.7 & 5.5 & & 3.3 & 3.7 & 9.9 & 11.6 & 32830 & 30200 & \\
\hline $\mathrm{H}_{3} \mathrm{CO}$ & $\mathrm{CN}$ & 17 & 31 & 38 & 56 & 58 & 5.9 & 5.5 & 4.5 & 4.6 & 4.7 & 10.1 & 11.8 & 31940 & 29510 & $27780^{a}$ \\
\hline $\mathrm{Me}_{2} \mathrm{~N}$ & $\mathrm{CN}$ & 18 & 34 & & 59 & 60 & 7.2 & 6.9 & & 7.0 & 6.7 & 10.2 & 11.9 & 31130 & 28840 & \\
\hline $\mathrm{H}$ & $\mathrm{NO}_{2}$ & 16 & 30 & & 57 & 58 & 8.1 & 7.5 & & 9.5 & 13.6 & 9.3 & 10.6 & 31780 & 28060 & \\
\hline $\mathrm{H}_{3} \mathrm{CO}$ & $\mathrm{NO}_{2}$ & 17 & 33 & 40 & 56 & 64 & 8.2 & 7.5 & 4.8 & 9.5 & 13.8 & 9.7 & 10.9 & 31140 & 27510 & $\begin{array}{l}25190^{a} \\
24880^{d, e}\end{array}$ \\
\hline $\mathrm{Me}_{2} \mathrm{~N}$ & $\mathrm{NO}_{2}$ & 17 & 35 & 40 & 64 & 73 & 9.6 & 8.9 & 7.6 & 12.6 & 15.0 & 9.8 & 11.2 & 30090 & 26920 & $\begin{array}{l}25770^{d, f} \\
22620^{a} \\
21980^{d, e} \\
19690^{d, f}\end{array}$ \\
\hline
\end{tabular}

${ }^{a}$ From ref 62 unless otherwise noted. Absorption in $\mathrm{CHCl}_{3} .{ }^{b}$ See ref $60 .{ }^{c}$ See ref 63 in gas phase. ${ }^{d}$ From ref $4 .{ }^{e}$ In DMF solution. ${ }^{f}$ In dioxane solution.

TABLE 3: BOA and Dipolar Properties of Selected D/A Polyenes

\begin{tabular}{|c|c|c|c|c|c|c|c|c|}
\hline \multirow[b]{2}{*}{ molecule } & \multicolumn{2}{|c|}{ BOA } & \multicolumn{2}{|c|}{$\Delta \mu(\mathrm{D})$} & \multicolumn{2}{|c|}{$\Delta \alpha\left(\AA^{3}\right)$} & \multicolumn{2}{|c|}{$\alpha_{\mathrm{g}}$ long axis $\left(\AA^{3}\right)$} \\
\hline & this work & literature & this work $^{e}$ & literature & this work $^{e}$ & literature & this work ${ }^{e}$ & literature \\
\hline DAO & $\begin{array}{l}-0.47^{a} \\
-0.07^{a}\end{array}$ & $\begin{array}{l}-0.47^{d} \\
-0.07^{d}\end{array}$ & $\begin{array}{r}12 \\
7\end{array}$ & $\begin{array}{r}\sim 14^{d} \\
\sim 3^{d}\end{array}$ & & & $\begin{array}{r}69 \\
110\end{array}$ & $\begin{array}{r}\sim 40^{d} \\
\sim 120^{d}\end{array}$ \\
\hline $\begin{array}{l}\text { AHTA } \\
\text { CAHT }\end{array}$ & $\begin{array}{l}-0.52^{b} \\
-0.39^{b} \\
-0.07^{c}\end{array}$ & $\begin{array}{l}-0.5^{f} \\
-0.1^{f}\end{array}$ & $\begin{array}{l}9.7 \\
8.1 \\
5.7\end{array}$ & $10^{f} 1.6^{f}$ & $\begin{array}{r}10 \\
4 \\
-1\end{array}$ & $\begin{array}{r}550^{f} \\
-120^{f}\end{array}$ & & \\
\hline
\end{tabular}

${ }^{a} \mathrm{C}-\mathrm{C}$ bond lengths same as those reported in ref $1 .{ }^{b}$ Geometries optimized with AM1. ${ }^{c}$ We used bond lengths of $1.433,1.365,1.405,1.383$, 1.391, and $1.389 \AA$ starting from the acceptor end of the molecule. The BLA is $0.011 .{ }^{d}$ Calculated using the INDO/SDCI/SOS method. See ref 1 . ${ }^{e}$ Using INDO1s/SCI/FF method. ${ }^{f}$ Measured by electroabsorption spectroscopy in frozen glasses of 2-methyltetrahydrofuran. See ref 54 .

by the electroabsorption measurement, and as a result, a much larger value of $\Delta \mu$ (Table 3 ).

The electroabsorption spectra of both of these molecules were measured in frozen glasses of 2-methyltetrahydrofuran. In an attempt to model the solvent effects on the spectrum, we first added an Onsager reaction-field correction ${ }^{55}$ which only increased the calculated values of $\Delta \alpha$ by about $10 \%$. The Onsager model applies a correction to the magnitudes of both $\Delta \mu$ and $\Delta \alpha$ due to the solvent field but does not account for possible changes in molecular geometry (such as BOA) that result from the interactions of the polyene with such solvent fields. ${ }^{57,58}$ For example, it has been reported that solvation has large effects on both BOA and the nonlinear optical properties of $\mathrm{D}-\mathrm{A}$ polyenes, ${ }^{1-3}$ and it has been observed that the Stark effect spectra of a D-A polyene in different solvents can yield very different values for $\Delta \alpha .^{53,54}$ This prompted us to alter the BOA of CAHT by changing the bond lengths so that the BOA was closer to that predicted by the experiment (Table 3). ${ }^{54}$ The anticipated trends between BOA, $\Delta \mu$ and $\Delta \alpha$ are followed, but the magnitudes of the $\Delta \alpha$ values are still very small. We are currently investigating whether other properties of the solvent (i.e., cavity size or rigidity) play a role in the analysis of Stark effect spectra. ${ }^{59}$

\section{Conclusions}

The SCI/FF and SDCI/SOS methods provide nearly identical results when used to calculate $\Delta \alpha$ of both polar and nonpolar polyenes, although the SCI/FF method more accurately predicts $\alpha_{\mathrm{g}}$. The shielding effect of phenyl groups on the D/A ability of substituents is significant. Calculations connecting BOA to $\Delta \alpha$ support Marder's theory that a smaller magnitude of BOA yields a smaller $\Delta \alpha$. However, our calculations show that neither solvent fields nor D/A substitution can entirely account for the large magnitudes of $\Delta \alpha$ measured by Stark-effect spectroscopy. 53,54
Acknowledgment. We thank Drs. David Yaron and JeanLuc Brédas for useful discussions and suggestions regarding this manuscript. We would also like to thank our sources of funding: The Winters Foundation, the Petroleum Research Fund administered by the American Chemical Society, and the NSFRPG and CAREER programs. The work in Mons is supported by the Belgium Prime Minister's Federal Service for Scientific, Technical and Cultural Affairs (Interuniversity Attraction Pole 4/11 in Supramolecular Chemistry and Catalysis).

\section{References and Notes}

(1) Meyers, F.; Marder, S. R.; Pierce, B. M.; Bredas, J. L. J. Am. Chem. Soc. 1994, 116, 10703-10714.

(2) Marder, S. R.; Gorman, C. B.; Meyers, F.; Perry, J. W.; Bourhill, G.; Bredas, J.-L.; Pierce, B. M. Science 1994, 265, 632-635.

(3) Zuliani, P.; DelZoppo, M.; Castiglioni, C.; Zerbi, G.; Marder, S. R.; Perry, J. W. J. Chem. Phys. 1995, 103, 9935-9940.

(4) Spangler, C. W.; Hall, T. J.; Saindon, M. L.; Rogers, R. D.; McCoy, R. K.; Birge, R. R.; Fleitz, P. A.; Zhang, C.-F. In Proceedings of the International Conference on Materials for Nonlinear and Electrooptics; Institute of Physics: Cambridge, England, 1989; pp 233-238.

(5) Di Bella, S.; Marks, T. J.; Ratner, M. A. J. Am. Chem. Soc. 1994 $116,4440-4445$.

(6) Albert, I. D. L.; Marks, T. J.; Ratner, M. A. J. Phys. Chem. 1996, $100,9714-9725$.

(7) Blanchard-Desce, M.; Wortmann, R.; Lebus, S.; Lehn, J.-M.; Kramer, P. Chem. Phys. Lett. 1995, 243, 526-532.

(8) The concept of BOA has been introduced by Marder's group to describe the trends observed in NLO properties of polyenes as a function of conjugation. The more conjugated the system, the smaller the magnitude of BOA. It is related to the average bond length alternation (BLA) between $\mathrm{C}-\mathrm{C}$ and $\mathrm{C}=\mathrm{C}$ bonds in a polyene, with a BLA of $0.11 \AA$ corresponding to a BOA of -0.6 . See ref 1 for more information.

(9) Oudar, J. L.; Chemla, D. S. J. Chem. Phys. 1977, 66, 2664-2668.

(10) Oudar, J. L. J. Chem. Phys. 1977, 67, 446-457.

(11) Levine, B. F.; Bethea, C. G.; Wasserman, E.; Leenders, L. J. Chem. Phys. 1978, 68, 5042-5045.

(12) Bishop, D. M.; Kirtman, B.; Champagne, B. J. Chem. Phys. 1997, 107, 5780-5787. 1007.

13) Wortmann, R.; Bishop, D. M. J. Chem. Phys. 1998, 108, 1001- 
(14) Beck, B.; Grummt, U.-W. J. Phys. Chem. B 1998, 102, 664-670.

(15) Barzoukas, M.; Muller, J.; Fort, A.; Marder, S. R.; Alain, V.; Blanchard-Desce, M. In Nonlinear Optical Properties of Organic Materials IX; Denver, CO, 1996; pp 132-141.

(16) Cammi, R.; Mennucci, B.; Tomasi, J. J. Phys. Chem. A 1998, 102, $870-875$.

(17) Liptay, W. In Modern Quantum Chemistry Part III: Action of Light and Organic Crystals; Sinanoglu, O., Ed.; Academic Press: 1965; pp 4566.

(18) Amos, A. T.; Burrows, B. L. Theor. Chim. Acta (Berlin) 1972, 23, $327-335$.

(19) Hurst, G. J. B.; Dupuis, M.; Clementi, E. J. Chem. Phys. 1988, 89, $385-395$.

(20) Marchese, F. T.; Jaffe, H. H. Theor. Chim. Acta (Berlin) 1977, 45, $241-247$.

(21) Kirtman, B.; Toto, J. L.; Robins, K. A.; Hasan, M. J. Chem. Phys. 1995, 102, 5350-5356.

(22) Cardelino, B. H.; Moore, C. E.; Frazier, D. O. J. Phys. Chem. A 1997, 101, 2207-2214.

(23) Sheng, Y.; Jiang, Y.; Wang, X.-C. J. Chem. Soc., Faraday Trans. 1998, 94, 47-52.

(24) Jonsson, D.; Norman, P.; Luo, Y.; Agren, H. J. Chem. Phys. 1996, $105,581-587$.

(25) Marder, S. R.; Beratan, D. N.; Cheng, L.-T. Science 1991, 252, $103-106$.

(26) Champagne, B.; Fripiat, J. G.; Andre, J.-M. J. Chem. Phys. 1992, $96,8330-8337$.

(27) Hudson, B. S.; Kohler, B. E.; Schulten, K. In Excited States; Lim, E.; Ed.; Academic Press: 1982; Vol. 6; pp 2.

(28) Ridley, J. E.; Zerner, M. Theor. Chim. Acta (Berlin) 1976, 42, 223-236.

(29) Ridley, J.; Zerner, M. Theor. Chim. Acta (Berlin) 1973, 32, 111134.

(30) Kurtz, H. A.; Stewart, J. J. P.; Dieter, K. M. J. Comput. Chem. 1990, 11, 82-87.

(31) Kurtz, H. A. Int. J. Quantum Chem. Symp. 1990, 24, 791-798.

(32) Meyer, H.; Schulte, K.-W.; Schweig, A. Chem. Phys. Lett. 1975, 31, 187-191.

(33) Locknar, S. A.; Peteanu, L. A. J. Phys. Chem. B 1998, 102, 42404246.

(34) Beljonne, D.; Shuai, Z.; Bredas, J. L. J. Chem. Phys. 1993, 98, 8819-8828.

(35) Shuai, Z.; Beljonne, D.; Bredas, J. L. J. Chem. Phys. 1992, 97, $1132-1137$

(36) Dewar, M. J. S.; Zoebisch, E. G.; Healy, E. F.; Stewart, J. J. P. J. Am. Chem. Soc. 1985, 107, 3902-3909.

(37) Coolidge, M. B.; Stewart, J. J. P. Technical Report. Seiler Research Laboratory, United States Air Force Academy, Colorado Springs, CO, 1990.

(38) Champagne, B.; Mosley, D. H.; Andre, J.-M. Int. J. Quantum Chem. Symp. 1993, 27, 667-685.
(39) Mataga, N.; Nishimoto, K. Z. Phys. Chem. 1957, 13, 140-157.

(40) Nishimoto, Z.; Mataga, N. Z. Phys. Chem. 1957, 12, 335-338.

(41) Tavan, P.; Schulten, K. J. Chem. Phys. 1986, 85, 6602-6609.

(42) Tavan, P.; Schulten, K. Phys. Rev. B. 1987, 36, 4337-4358.

(43) Ohno, K. Theor. Chem. Acta 1964, 2, 219-227.

(44) Klopman, G. J. Am. Chem. Soc. 1964, 86, 4550-4557.

(45) Luo, Y.; Agren, H.; Koch, H.; Jorgensen, P.; Helgaker, T. Phys. Rev. B. 1995, 51, 14949-14957.

(46) Rowe, D. J. Rev. Mod. Phys. 1968, 40, 153-166.

(47) Lasaga, A. C.; Karplus, M. J. Chem. Phys. 1979, 71, 1218-1234.

(48) Leopold, D. G.; Pendley, R. D.; Roebber, J. L.; Hemley, R. J.; Vaida, V. J. Chem. Phys. 1984, 81, 4218-4229.

(49) de Melo, C. P.; Silbey, R. J. Chem. Phys. 1988, 88, 2558-2566.

(50) One cause for concern was whether neglecting certain states in the $\mathrm{SCI} / \mathrm{FF}$ calculation would change our calculated values compared to a more rigorous (and expensive) MRD/SDCI/SOS method. The electronic energy levels of polyenes are characterized by a ground state of $\mathrm{A}_{\mathrm{g}}$ symmetry, a low-lying 2-photon allowed $2 \mathrm{~A}_{\mathrm{g}}$ state, a one-photon- allowed $1 \mathrm{~B}_{\mathrm{u}}$ state, a higher lying two-photon-allowed state commonly known as the $\mathrm{mA}_{\mathrm{g}}$ state, as well as many other higher-lying states. Using SCI, it is likely that the transition moment from the $1 \mathrm{~B}_{\mathrm{u}}$ to higher lying $\mathrm{A}_{\mathrm{g}}$ states would be underestimated which could have lead to smaller values of $\alpha_{e}$ than in the MRD/SDCI/SOS method. However, our calculations show that the results are not significantly affected by these possible omissions.

(51) Liptay, W. In Excited States; Lim, E. C., Ed.; Academic Press: New York, 1974; pp 129-229.

(52) Bublitz, G. U.; Boxer, S. G. Annu. Rev. Phys. Chem. 1997, 48, $213-242$

(53) Bublitz, G. U.; Ortiz, R.; Runser, C.; Fort, A.; Barzoukas, M.; Marder, S. R.; Boxer, S. G. J. Am. Chem. Soc. 1997, 119, 2311-2312.

(54) Bublitz, G. U.; Ortiz, R.; Marder, S. R.; Boxer, S. G. J. Am. Chem Soc. 1997, 119, 3365-3376.

(55) Onsager, L. J. Am. Chem. Soc. 1936, 58, 1486-1493.

(56) Marder, S. R.; Gorman, C. B.; Tiemann, B. G.; Cheng, L.-T. J. Am. Chem. Soc. 1993, 115, 3006-3007.

(57) Gao, J.; Alhambra, C. J. Am. Chem. Soc. 1997, 119, 2962-2963.

(58) Pappalardo, R. R.; Marcos, E. S.; Ruiz-Lopez, M. F.; Rinaldi, D.; Rivail, J.-L. J. Am. Chem. Soc. 1993, 115, 3722-3730.

(59) Locknar, S. A.; Chowdhury, A.; Premvardhan, L.; Peteanu, L. A. In preparation.

(60) Liptay, W.; Wortmann, R.; Bohm, R.; Detzer, N. Chem. Phys. 1988, 120, 439-448.

(61) Ponder, M. C. PhD Thesis, University of California, Berkeley, 1983.

(62) Cheng, L.-T.; Tam, W.; Marder, S. R.; Stiegman, A. E.; Rikken, G.; Spangler, C. W. J. Phys. Chem. 1991, 95, 10643-10652.

(63) Heimbrook, L. A.; Kohler, B. E.; Spiglanin, T. A. Proc. Natl. Acad. Sci. U.S.A. 1983, 80, 4580-4584. 